\title{
Modelagem Matemática e Interdisciplinaridade como Estratégia de Aprendizado Significativo: Solução de um Circuito em série RLC.
}

\author{
Ramos, G. B. G.; Gonzaga, B.R. \\ Coordenadoria de Engenharia de Controle e Automação, Instituto Federal do Espírito Santo, Serra, ES, Brasil.
}

\begin{abstract}
Resumo
O presente artigo tem como objetivo utilizando a modelagem matemática e a interdisciplinaridade para promover a aprendizagem significativa do conteúdo de Equações Diferenciais. A modelagem e solução de um circuito são de grande interesse para alunos dos cursos de Engenharia de Controle e Automação e de Engenharia Elétrica. A apresentação de problemas que envolvam esse assunto, em um curso de Equações Diferenciais, desperta uma grande motivação por parte do aluno para o aprendizado da teoria matemática necessária para a sua solução. A proposta desse artigo é apresentar a modelagem matemática para um circuito em série RLC, que resulta em uma Equação Diferencial Ordinária (EDO), e solucioná-lo de diferentes maneiras à luz da teoria de Circuitos. Os métodos utilizados para encontrar a solução desse modelo foram a Teoria de Equações Diferenciais de Ordem Superior, e a Teoria de Transformada de Laplace. Não faz parte do objetivo desse trabalho fazer uma análise profunda dos métodos de Equações Diferenciais Ordinárias que foram empregados nas soluções do problema proposto.
\end{abstract}

Palavras chaves: modelagem matemática, equações diferenciais ordinárias, circuito em séria RLC, aprendizado significativo, interdisciplinaridade.

\begin{abstract}
This article aims at using mathematical modeling and interdiciplinaridade to promote meaningful learning of Differential Equations content. The modeling and solution of a circuit are of great interest to students of Control and Automation Engineering, and Electrical Engineering courses as well. The presentation of problems involving this subject in a course of Differential Equations, arouses a great motivation for the student to learn the mathematical theory required for its solution. The purpose of this paper is to present the mathematical modeling for a series RLC circuit, resulting in an ordinary differential equation (ODE) and solve it in different ways in the light of circuits theory. The methods used to find the solution of this model were the Equation Theory Higher Order differentials, and the Theory of Laplace Transform. It is not the aim of this study do a thorough analysis of the methods of ordinary differential equations that were used in the solutions of the proposed issue.
\end{abstract}

Keywords: Mathematical modeling, ordinary differential equations, RLC series circuit, meaningful learning, interdisciplinary.

\section{Introdução}

O surgimento da ideia de utilizar a modelagem matemática como ferramenta educacional para promover o aprendizado significativo segundo Biembengut [1] teve seu apogeu internacional na década de 60 sob o ideal de aplicar os conhecimentos matemáticos para solucionar problemas das ciências em geral. Biembengut [1] destaca que no Brasil, esse movimento ocorre praticamente ao mesmo tempo que no cenário internacional, já nas décadas de 70 e 80 há o surgimento de vários trabalhos e grupos de pesquisa que conquistaram adeptos por todo o país. Como se faz um modelo matemático e como empregá-lo no ensino da matemática é um dos assuntos de interesse desses grupos. No entanto, a modelagem matemática 
só se transforma em ferramenta de aprendizado se direcionada ao assunto de interesse para o aluno, nesse contexto surge a interdisciplinaridade, um tema cada vez mais presente nos cursos de Engenharia.

Segundo Japiassu [2], a interdisciplinaridade é uma organização cooperativa e coordenada do ensino, que possibilita a cooperação mútua entre diferentes disciplinas. Essa prática de ensino é motivadora e desafiadora, para alunos e professores. Do ponto de vista do professor, a interdisciplinaridade propicia um ambiente de contínua aprendizagem, assim como a troca de experiências sobre metodologias de ensino entre os professores do curso. Do ponto de vista do aluno, essa prática de ensino é uma oportunidade de um primeiro contato com a linguagem e os problemas das disciplinas técnicas do curso.

Nesse contexto cria-se um ambiente favorável à aprendizagem significativa. Segundo Borssoi [3] a aprendizagem significativa ocorre quando a apresentação de problemas de interesse do aluno, que privilegie os conhecimentos pré-existentes em sua estrutura cognitiva, gera uma pré-disposição ao aprendizado.

Para o curso de Engenharia de Controle e Automação a escolha da interdisciplinaridade entre Equações Diferenciais e Circuitos é uma forma de promover a aprendizagem significativa. A disciplina de Circuitos possui elementos pré-existentes na estrutura cognitiva dos alunos desde o ensino médio. Além disso, ela possui grande importância para diversas disciplinas desse curso, propiciando a pré-disposição ao aprendizado. Sendo assim, trabalhar no curso de Equações Diferenciais, a modelagem matemática para um problema de Circuitos, proporciona um aprendizado significativo dos conteúdos preteridos dessa disciplina. Nesse contexto, o presente trabalho elege o circuito em série RLC para se trabalhar a modelagem matemática. Essa escolha se deve não somente ao citado anteriormente, como também por se tratar de um problema que possibilita diferentes abordagens para sua solução. Dessa forma o aluno desenvolve uma postura crítica para solução de problemas.

\section{Apresentação do Problema}

Na Figura 1 são apresentados os elementos de um circuito elétrico. Ao analisar essa figura, os alunos do curso de Engenharia, devido aos conteúdos de Física do ensino médio, reconhecem seus elementos: o resistor, o capacitor, o indutor e o gerador. Algumas das relações matemáticas presentes na Figura 1 são relacionadas com o curso de Cálculo Diferencial do ensino superior, como acontece com a relação para o indutor entre a queda de tensão e a corrente. Dessa forma a análise dessa figura dá início ao processo cognitivo desejado.

Diferentes tipos de circuito poderiam ser montados relacionando os componentes da Figura 1, porém 0 escolhido é o circuito de malha fechada em série RLC, como mostra a Figura 2. Esse circuito foi escolhido por conter todos os elementos da Figura 1 de uma forma simplificada.

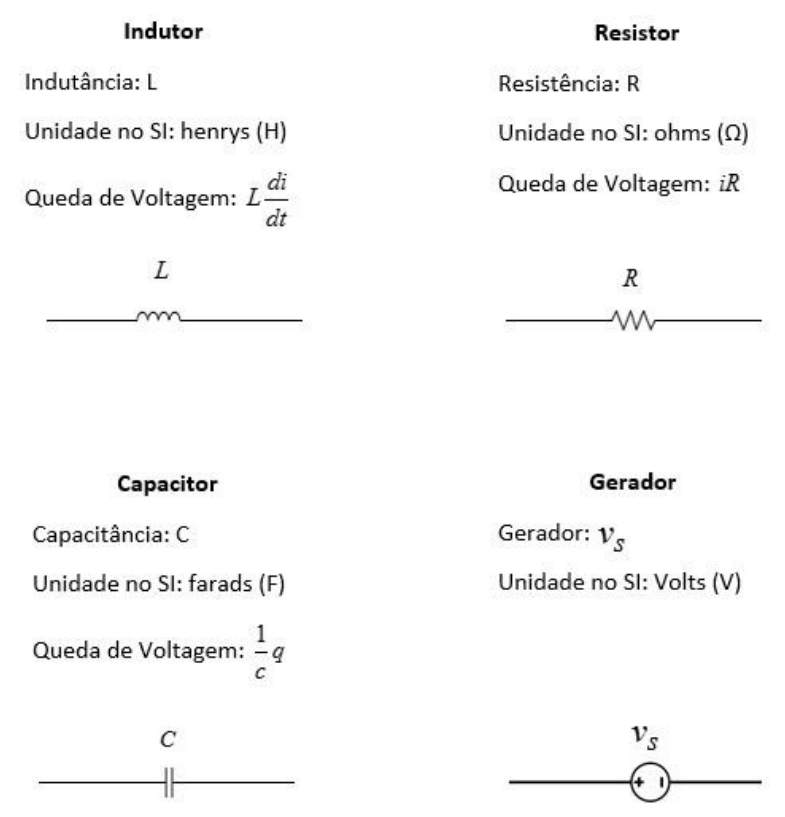

Figura 1: Elementos de um circuito

O processo de modelagem do circuito da Figura 2 possui os seguintes objetivos: introduzir a linguagem utilizada na disciplina de circuitos, introduzir novos conceitos necessários à solução do problema, e ainda, se necessário, retomar conteúdos de matemática para o completo entendimento da modelagem do problema. Segundo Dullius et all [4] a falta de conteúdos básicos de matemática, e a dificuldade de aceitação de funções como solução de um problema, é um dos fatores da não compreensão da disciplina de Equações Diferenciais.

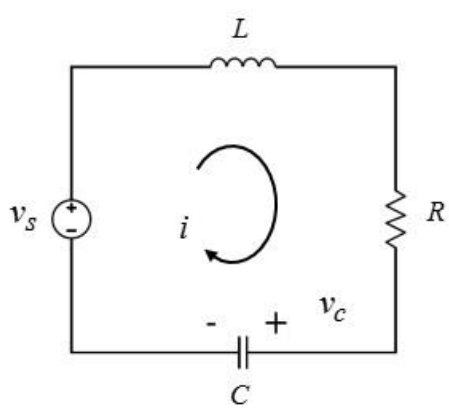

Figura 2: Circuito em série RLC

A modelagem matemática para esse circuito pode ser apresentada de várias maneiras, dependendo do que se deseja saber sobre o problema. Nesse momento há a necessidade de recorrer à teoria de Circuitos para descobrir quais são as variáveis de interesse para o circuito ou, na linguagem da teoria de Circuitos, as 
saídas do circuito. As variáveis de saída para o circuito da Figura 2 são: a corrente no indutor, e a tensão no capacitor. Essas variáveis são denominadas Variáveis de Estado do sistema.

O presente trabalha irá desenvolver a modelagem matemática do problema utilizando a segunda Lei de Kirchhoff. Essa lei diz que a voltagem aplicada em uma malha fechada deve ser igual à soma das quedas de voltagem na malha. Dessa forma encontra-se uma Equação Diferencial Ordinária (EDO) de segunda ordem que modela o circuito da Figura 2. Segundo Dorf et all [5] a ordem da equação diferencial que representa um circuito é no máximo a soma do número de capacitores com o número de indutores.

Para contextualizar o modelo matemático encontrado é proposto um caso particular desse circuito, conforme mostra a Figura 3.

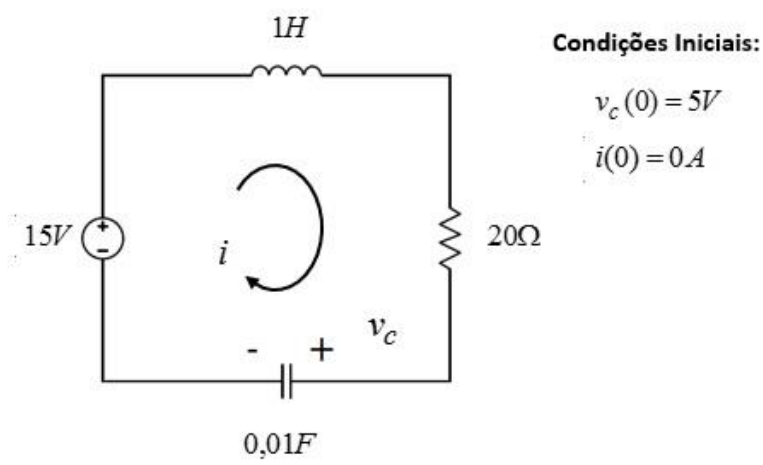

Figura 3: Circuito em série RLC particular.

$\mathrm{Na}$ Figura 3 são dadas as entradas do circuito: a tensão no gerador, a indutância, a resistência e a capacitância. O gerador é de tensão contínua, são exemplos de geradores de tensão contínua as baterias e pilhas, a tensão nas redes elétricas é modelada por uma senóide. São dadas também as condições iniciais do sistema, o papel dessas condições será discutido ao se resolver o problema em questão.

\section{Modelagem e Solução}

Aplicando a segunda lei de Kirchhoff ao circuito da Figura 2, obtém-se a Equação Diferencial Ordinária (EDO):

$$
\mathrm{L} \frac{\mathrm{di}(\mathrm{t})}{\mathrm{dt}}+\mathrm{Ri}(\mathrm{t})+\frac{1}{\mathrm{C}} \mathrm{q}(\mathrm{t})=\mathrm{v}_{\mathrm{s}}(\mathrm{t})
$$

Para uma análise numérica do modelo matemático descrito na Equação (1) será estudado o caso particular da Figura 3 de três formas.

Em uma primeira forma de solução para o circuito RLC da Figura 2, será considerada a relação da corrente com a carga no capacitor conforme Equação (2).

$$
\mathrm{i}(\mathrm{t})=\frac{\mathrm{dq}(\mathrm{t})}{\mathrm{dt}}
$$

O intuito dessa relação é transformar a EDO da Equação (1) em uma EDO Linear de segunda ordem. A substituição da Equação (2) na Equação (1) apresenta um modelo matemático para o circuito em série RLC da Figura 2 de uma EDO Linear de segunda ordem em termos da carga do capacitor.

$$
L \frac{d^{2} q(t)}{d t^{2}}+R \frac{q(t)}{d t}+\frac{1}{C} q(t)=v_{s}(t)
$$

Para o circuito da Figura 3, a Equação (3) resulta em uma EDO Linear de segunda ordem com coeficientes constantes que será resolvido de acordo com Boyce et all [6].

$$
\frac{\mathrm{d}^{2} \mathrm{q}(\mathrm{t})}{\mathrm{dt}^{2}}+20 \frac{\mathrm{dq}(\mathrm{t})}{\mathrm{dt}}+100 \mathrm{q}(\mathrm{t})=15
$$

A solução da Equação (4) é a soma da solução da equação homogênea $q_{h}(t)$ associada à Equação (4), que na teoria de Circuito é chamada de resposta natural (não forçada) de um circuito, com a solução particular para a equação $q_{p}(t)$, que na teoria de Circuitos é chamada de resposta forçada do circuito, portanto:

$$
q(t)=q_{h}(t)+q_{p}(t)
$$

Aplicando o método de solução que trata das Equações Lineares Homogêneas com coeficientes constantes, a resposta natural do sistema representado pela equação (6) é da forma $q_{h}(t)=e^{m t}$.

$$
\begin{aligned}
& \frac{\mathrm{d}^{2} \mathrm{q}_{\mathrm{h}}(\mathrm{t})}{\mathrm{dt^{2 }}}+20 \frac{\mathrm{dq} \mathrm{q}_{\mathrm{h}}(\mathrm{t})}{\mathrm{dt}}+100 \mathrm{q}_{\mathrm{h}}(\mathrm{t})=0 \\
& \left(\mathrm{~m}^{2}+20 \mathrm{~m}+100\right) e^{m t}=0
\end{aligned}
$$

A solução para a Equação (7) são duas raízes reais iguais a $m=-10$. Para a teoria de Circuitos há uma classificação do sistema de acordo com as raízes da equação em m. Segundo Dorf et all [5] quando as raízes são iguais, esse sistema é denominado criticamente amortecido, para raízes reais distintas o sistema é dito superamortecido e finalmente para raízes complexas o sistema é dito subamortecido.

Considerando $k_{1}$ e $k_{2}$ constantes a solução natural para o sistema é dada por:

$$
q_{h}(t)=k_{1} e^{-10 t}+t k_{2} e^{-10 t}
$$

A solução particular, ou solução forçada, para o sistema é uma constante, portanto $q_{p}(t)=k_{a}$ aplicada à solução particular na Equação (4) resulta:

$$
100 q_{p}(t)=15 \Rightarrow q_{p}(t)=0,15
$$


A solução geral para o sistema é dada pela Equação (10).

$$
\mathrm{q}(\mathrm{t})=\mathrm{k}_{1} e^{-10 \mathrm{t}}+t \mathrm{k}_{2} e^{-10 t}+0,15
$$

Uma das condições iniciais do sistema é $v_{c}(0)=5$.

$$
\frac{\mathrm{q}(\mathrm{t})}{\mathrm{C}}=\mathrm{v}_{\mathrm{c}}(\mathrm{t}) \Rightarrow \frac{\mathrm{q}(0)}{\mathrm{C}}=\mathrm{v}_{\mathrm{d}}(0) \Rightarrow k_{1}=-0,1
$$

Ao utilizar as Equações (2) e (10) encontra-se:

$$
i(t)=\left(-10 k_{1}+k_{2}\right) e^{-10 t}-10 k_{2} e^{-10 t}
$$

A condição inicial $\tilde{\mathrm{i}}(0)=0$ juntamente com as Equações (11) e (12) resulta:

$$
k_{2}=-1
$$

Substituindo as Equações (11) e (13) na Equação (10) encontra-se a solução para o circuito em série RLC da Figura 2.

$$
\mathrm{q}(\mathrm{t})=-0,1 e^{-10 t}-t e^{-10 t}+0,15
$$

Do ponto de vista da teoria de Equações Diferencias o problema está modelado e solucionado, porém para a teoria de Circuitos uma análise do sistema da Figura 2, através da carga do capacitor, não é interessante. Em circuitos as variáveis de interesse, chamadas Variáveis de Estado, são a tensão no capacitor $v_{c}$ e a corrente no indutor î. Um fato interessante é que a corrente no indutor é dada pela Equação (12), se as condições iniciais do sistema não fossem conhecidas, não seria possível o cálculo de $k_{1}$ e $k_{2}$, dessa forma a solução do circuito da Figura 3 seria uma família de funções como mostra a Figura 4.

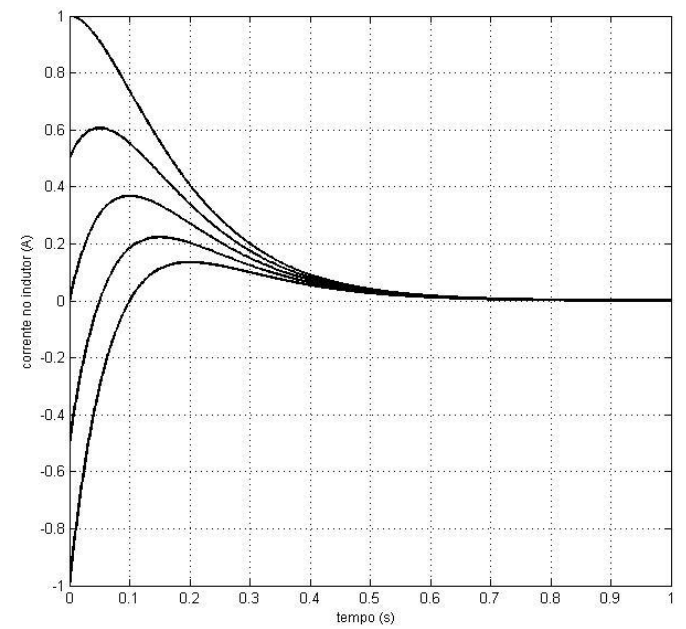

Figura 4: Relação da corrente no indutor com o tempo, sem o conhecimento das condições iniciais do problema.
Para fazer os gráficos da Figura 4 considerou-se $k_{2}=-1$ e variou-se $0 k_{1}$.

A equação da tensão no capacitor, dadas as condições iniciais da Figura 3, é encontrada utilizando-se a relação $v_{c}(t)=\frac{q(t)}{c}$, da Figura 1 , juntamente com as Equações (10), (11) e (13). O gráfico da tensão do capacitor com o tempo está representado na Figura 6.

$$
v_{c}(t)=-10 e^{-10 t}-100 t e^{-10 t}+15
$$

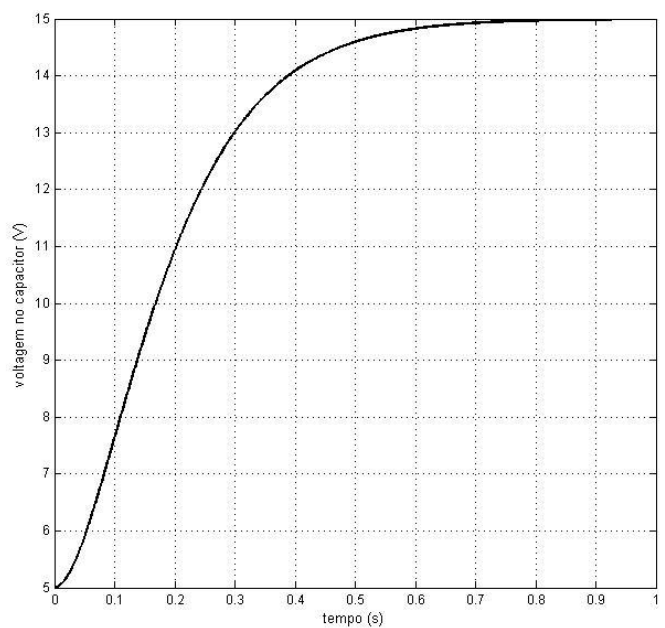

Figura 5: Relação da tensão no capacitor com o tempo, dadas as condições iniciais do sistema.

Dadas às condições iniciais do sistema da Figura 3 , as Equações (11), (12) e (13) fornecem a equação da corrente no indutor, cujo gráfico está representado na Figura 5.

$$
i(t)=10 \mathrm{t} e^{-10 t}
$$

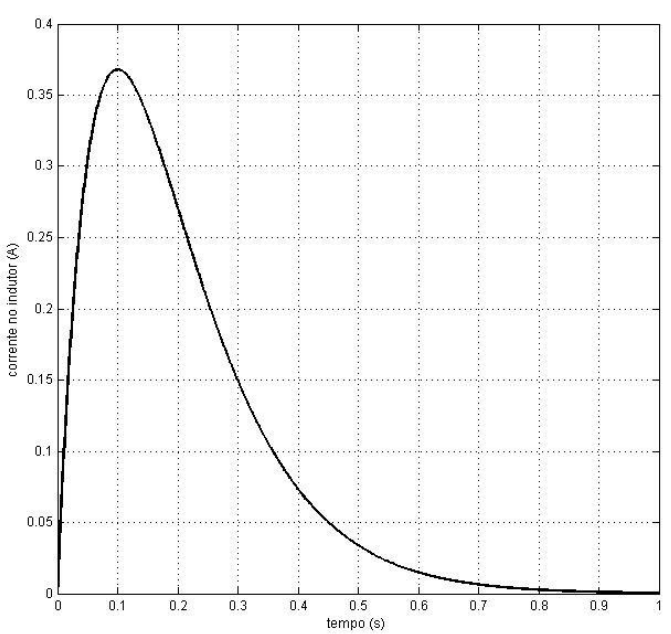

Figura 6: Relação da corrente no indutor com o tempo, dadas as condições iniciais do sistema. 
Portanto, as condições iniciais do sistema são importantes para especificar qual, dentre as funções encontradas, informa as condições do problema com o passar do tempo. Uma análise dos gráficos contidos na Figura 5 e Figura 6 fornece informações sobre 0 circuito RLC da Figura 3. Nota-se que a corrente no indutor, que inicialmente era nula, atinge um máximo próximo a 0,4 ampere e volta a ser nula. A tensão no capacitor, que inicialmente era de 5 volts atinge os 15 volts do gerador.

Em uma segunda forma de solução para o circuito RLC da Figura 2 será considerada a relação da corrente no indutor com a carga no capacitor integrando-se a Equação (2), para obter a Equação (17), onde $k_{4}$ é uma constante.

$$
\mathrm{q}(\mathrm{t})=k_{4}+\int_{0}^{\mathrm{t}} \mathrm{i}(\tau) d \tau
$$

A substituição da Equação (16) na Equação (1) apresenta um modelo matemático para o circuito em série RLC da Figura 2 de uma EDO íntegro-diferencial que será resolvido de acordo com Boyce et all [6].

$$
\mathrm{L} \frac{\mathrm{di}(\mathrm{t})}{\mathrm{dt}}+\operatorname{Ri}(\mathrm{t})+\frac{1}{\mathrm{C}}\left(k_{4}+\int_{0}^{\mathrm{t}} \mathrm{i}(\tau) d \tau\right)=v_{s}(t)
$$

Para o circuito da Figura 3, a Equação (18) tem a forma.

$$
\frac{\mathrm{di}(\mathrm{t})}{\mathrm{dt}}+20 \mathrm{i}(\mathrm{t})+5+100 \int_{0}^{\mathrm{t}} \mathrm{i}(\tau) d \tau=15
$$

O valor da constante $k_{4}=0,05$ foi encontrado utilizando-se $t=0$ nas equações (14) e (17).

Para a solução da Equação (19) utiliza-se a teoria de Convolução e Transformada de Laplace.

$$
\mathrm{sI}(\mathrm{s})-\mathrm{I}(0)+20 \mathrm{I}(\mathrm{s})+5+100 \frac{\mathrm{I}(\mathrm{s})}{\mathrm{s}}=15
$$

Considerando $\mathrm{i}(0)=0$ na Equação (19).

$$
\left(s^{2}+20 s+100\right) I(s)=10 \Rightarrow I(s)=\frac{10}{(g+10)^{2}}
$$

De uma forma direta e rápida obtém-se a Equação (16) para a corrente no indutor. Para encontrar a equação da tensão no capacitor é necessário utilizar a relação:

$$
v_{c}(t)=\frac{1}{c} \int_{0}^{t} \tilde{I}(\tau) d \tau
$$

Ao integrar a Equação (22) e utilizar a condição inicial $v(0)=5$ encontra-se a Equação (15) para a tensão no capacitor.

Nas duas primeiras soluções apresentadas para resolver a EDO que modela o circuito em série RLC, foi necessário lançar mão de mais de um processo matemático para adquirir as equações das duas variáveis de interesse.

Em uma terceira e última forma de solução para o circuito RLC da Figura 2, será considerado o modelo matemático de um sistema de EDO lineares de primeira ordem. A vantagem da utilização desse método é de que ao final do processo têm-se direto as duas equações das Variáveis de Estado do circuito.

$$
\left\{\begin{array}{c}
\mathrm{L} \frac{\mathrm{di}(\mathrm{t})}{\mathrm{dt}}+\operatorname{Ri}(\mathrm{t})+v_{c}(\mathrm{t})=v_{s}(t) \\
C \frac{d v_{d}(t)}{d t}=i(t)
\end{array}\right.
$$

Para a solução do sistema de EDO em (23) utiliza-se a teoria de Transformada de Laplace juntamente com as condições iniciais do sistema, $v_{c}(0)=5$ e $i(0)=0$, para obter:

$$
\left\{\begin{array}{l}
s(s+20) I(s)+s V_{c}(s)=15 \\
-I(t)+0,01 s V_{d}(s)=0,05
\end{array}\right.
$$

Para a solução do sistema em (24) utiliza-se a Regra de Cramer.

$$
\begin{aligned}
& D=\left|\begin{array}{cc}
s(s+20) & s \\
-1 & 0,01 s
\end{array}\right|=0,01 s(s+10)^{2} \\
& D_{I}=\left|\begin{array}{cc}
15 & s \\
0,05 & 0,01 s
\end{array}\right|=0,1 s \\
& D_{V_{c}}=\left|\begin{array}{cc}
s(s+20) & 15 \\
-1 & 0,05
\end{array}\right|=0,05 s^{2}+s+15 \\
& \mathrm{I}(s)=\frac{D_{1}}{D}=\frac{10}{(s+10)^{2}} \\
& V_{c}(s)=\frac{D_{V_{c}}}{D}=-\frac{10}{s+10}-\frac{100}{(s+10)^{2}}+\frac{15}{s}
\end{aligned}
$$

Utilizando-se a técnica de frações, obtém-se a Equação (29). Ao aplicar o Laplaciano inverso nas Equações (28) e (29) obtém-se de forma direta a Equação (15) da tensão no capacitor e a Equação (16) para a corrente no indutor.

\section{Conclusão}

O trabalho com modelagem matemática, na disciplina de Equações Diferenciais, é uma prática presente inclusive em algumas bibliografias básicas para a disciplina. Porém, trabalhar a modelagem matemática sem o cuidado de abordar um problema de interesse para o aluno, não é uma prática que favorece o aprendizado significativo. Essa prática de ensino, quando bem desempenhada, deixa evidente ao aluno a necessidade de se entender os conceitos de Equações Diferenciais para 0 entendimento de teorias importantes da sua formação. 
Ao se trabalhar com a interdisciplinaridade, o aluno sente-se motivado a encontrar a solução para o problema. É importante para esse processo o fomento superior. Assim, em algum momento, o aluno sentirá a necessidade de adquirir novos conceitos para solucionar, ou conseguir de forma mais rápida e adequada a solução que deseja.

Ao final desse processo, o aluno sente-se motivado. Um dos fatores para essa motivação é conseguir solucionar um problema de interesse para a sua formação, em uma disciplina do núcleo básico de sua formação. Nesse cenário o aluno percebe que aprendeu, pois ocorre um processo de relação e criação de estruturas cognitivas possibilitando assim a aprendizagem significativa. A partir dessa experiência, que ele entende como favorável, há a formação de uma postura crítica em relação ao que Ihe será apresentada a partir daí.

Enfim, a disciplina de Equações Diferenciais, em qualquer curso superior, possui um grande potencial para o desenvolvimento de trabalho semelhante. No entanto, para que essa prática se transforme em uma ferramenta pedagógica na promoção do aprendizado significativo, é necessário estar atento aos componentes curriculares do respectivo curso e principalmente aos interesses dos alunos.

\section{Referência}

de diferentes ideias para modelar e resolver o problema, privilegiando conhecimentos adquiridos ao longo de sua formação, seja no ensino médio ou [1] BIEMBENGUT, M. S. 30 Anos de Modelagem Matemática na Educação Brasileira: das propostas primeiras às propostas atuais. ALEXANDRIA Revista de Educação em Ciência e Tecnologia v.2, n.2, p. 7-32, jul 2009. Disponível em: http://alexandria.ppgect.ufsc.br/ acesso em: 10 de fevereiro 2015

[2] JAPIASSU, H. Interdisciplinaridade e patologia do saber. Rio de Janeiro: Imago, 1976. 74p.

[3] BORSSOI, A. D.; ALMEIDA, L. M. W. Modelagem matemática e aprendizagem significativa: uma proposta para o estuda das equações diferenciais ordinárias. Educação Matemática e Pesquisa. v.6, n.2, 2004. Disponível:

http://revistas.pucsp.br/index.php/emp/article/viewArticl e/4689

[4] DULliUS, M. M.; VEIT E. A.; ARAUJO, I. S. Dificuldades dos Alunos na Aprendizagem de Equações Diferenciais Ordinárias. ALEXANDRIA Revista de Educação em Ciência e Tecnologia, v.6, n.2 p. 207-228, junho 2013.

[5] DORF, R. C.; SVOBODA, J. A. Introdução aos Circuitos Elétricos $7^{a}$ edição. Rio de Janeiro: LTC: 2008.

[6] BOYCE, W E.; DIPRIMA, R. C. Equações Diferenciais Elementares e problemas de valores de contorno 9aㅡ edição. Rio de Janeiro: LTC: 2008. 